\title{
Cardiac Amyloidosis Presumptively Diagnosed as Cardiac Syndrome $\mathbf{X}$
}

\author{
Atsuhiko Yagishita, MD; Shuzou Tanimoto, MD; Kengo Tanabe, MD; \\ Akihiro Isogawa, MD*; Makoto Taniguchi, MD**; \\ Teruo Shiba, MD*; Kazuhiro Hara, MD
}

\begin{abstract}
A 75-year-old man with cardiac amyloidosis was presumptively diagnosed as having cardiac syndrome X. Early clinical presentation was repeated episodes of angina pectoris without angiographically significant stenosis of the coronary arteries, so the diagnosis of cardiac syndrome $\mathrm{X}$ was initially given. However, his anginal symptoms worsened despite appropriate medication. He developed congestive heart failure and died 8 years after his first anginal symptoms. Autopsy revealed amyloid deposits in the intramyocardial coronary arteries with luminal obstruction, with little in the epicardial coronary arteries or myocardium. Therefore, the definitive diagnosis was cardiac amyloidosis. (Circ J 2009; 73: 1349-1351)
\end{abstract}

Key Words: Cardiac amyloidosis; Cardiac syndrome X

$\mathbf{T}$ he most common clinical presentation of cardiac amyloidosis is congestive heart failure because of the restrictive cardiomyopathy caused by extensive interstitial infiltration of amyloid. Although typical symptoms of angina pectoris occasionally occur as the initial disease presentation, ${ }^{1-7}$ in most cases coronary angiography shows no significant stenosis of coronary arteries because amyloid deposits primarily in the intramyocardial coronary arteries rather than the epicardial coronary arteries? Therefore, it is difficult to accurately diagnose cardiac amyloidosis in its early clinical stages.

\section{Case Report}

In December 2000, a 69-year-old Japanese man, who had been treated for pyoderma gangrenosum, was referred to the cardiology department from a dermatologist for the purpose of evaluating his 2-year history of exertional angina. Because the exercise treadmill test showed STsegment depression on the electrocardiogram (Figure 1), he was suspected of having coronary artery disease. Left ventricular function was well preserved with no wall motion abnormalities as assessed by echocardiography. Coronary angiography showed no significant stenosis of any of the epicardial coronary arteries. The provocation test with ergonovine was performed to rule out coronary vasospasm, and a negative result was obtained (Figure 2). Therefore, the patient was diagnosed as having cardiac syndrome X. Despite intensive medical therapy with maximum doses of isosorbide mononitrate, nicorandil and cilnidipine (calciumchannel blocker), his anginal symptoms worsened. Repeated

(Received May 28, 2008; revised manuscript received July 5, 2008; accepted July 23, 2008; released online December 27, 2008)

Division of Cardiology, *Division of Internal Medicine and **Division of Gastroenterology, Mitsui Memorial Hospital, Tokyo, Japan

Mailing address: Shuzou Tanimoto, MD, Division of Cardiology, Mitsui Memorial Hospital, 1 Kanda-Izumi-cho, Chiyoda-ku, Tokyo 101-8643, Japan. E-mail: shuzou_bushido@msn.com

All rights are reserved to the Japanese Circulation Society. For permis-

sions, please e-mail: cj@j-circ.or.jp coronary angiography was performed in 2002, 2003 and 2004 , but did not show any significant stenosis of the coronary arteries.

During the 6-year observational period, his serum albumin concentration gradually decreased (from 4.2 to $2.2 \mathrm{~g} / \mathrm{dl}$ ). Because the patient did not have severe proteinuria or malnutrition, gastrointestinal malabsorption was suspected as the cause. Upper gastrointestinal endoscopy with gastric and duodenal biopsies was performed and amyloid deposits were detected in the tissue samples, so the diagnosis of gastrointestinal amyloidosis was established. In order to investigate the presence of systemic amyloidosis, the serum immunoglobulin component was assessed. Although serum IgA was elevated $(953 \mathrm{mg} / \mathrm{dl})$, serum IgG was suppressed $(418 \mathrm{mg} / \mathrm{dl})$, and electric plasma pheresis showed monoclonal gammopathy, comprising IgA-ramda type. A large amount of Benz-Jones protein was also detected in his urine. On the basis of these findings, the diagnosis of systemic amyloidosis was made and therefore, cardiac involvement of systemic amyloidosis was suspected as the cause of his anginal symptoms.

In December 2006, the patient was admitted for treatment of the pyoderma gangrenosum. At this time, rest angina was occurring frequently. He developed congestive heart failure and hemodynamic instability because of severe sepsis caused by cellulitis at the ulcerative site of pyoderma gangrenosum. Although mechanical respiratory support was instituted with an infusion of norepinephrine to maintain systemic hemodynamic status, he developed septic shock and ultimately died.

Autopsy revealed immunoglobulin light-chain-related amyloid deposits within the walls of systemic arteries. The large number of myeloid cells increased in bone marrow, which suggested the existence of secondary amyloidosis because of multiple myeloma (IgA-ramda type/AL amyloidosis) (Figure 3A). The cardiac amyloid deposits were observed primarily in the intramyocardial arteries with either luminal stenosis or occlusion (Figure 3B), which may have been the cause of scattered small infarcts in the myocardium. There was little amyloid deposit in the epicardial 
A
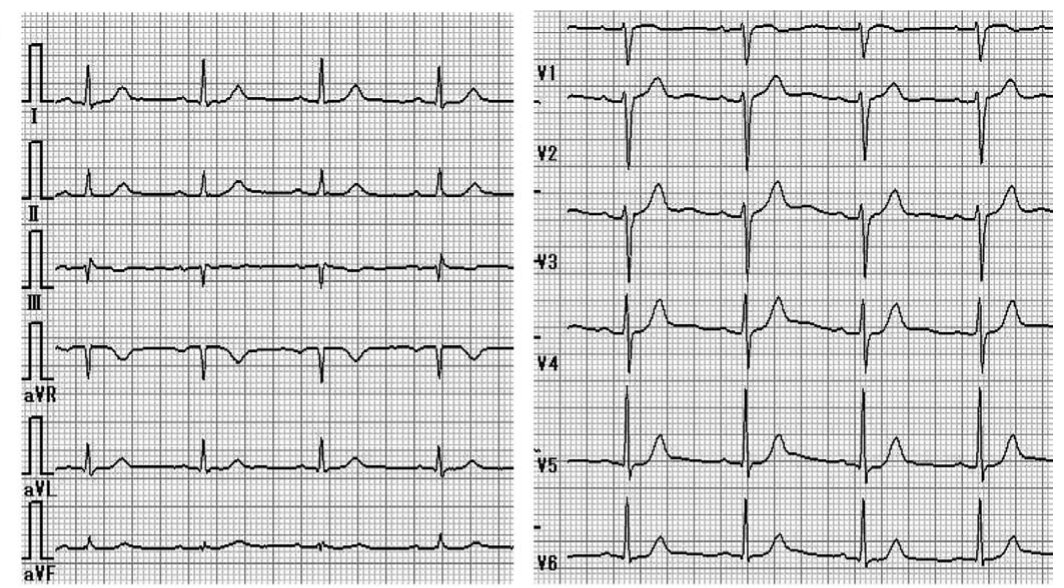

B
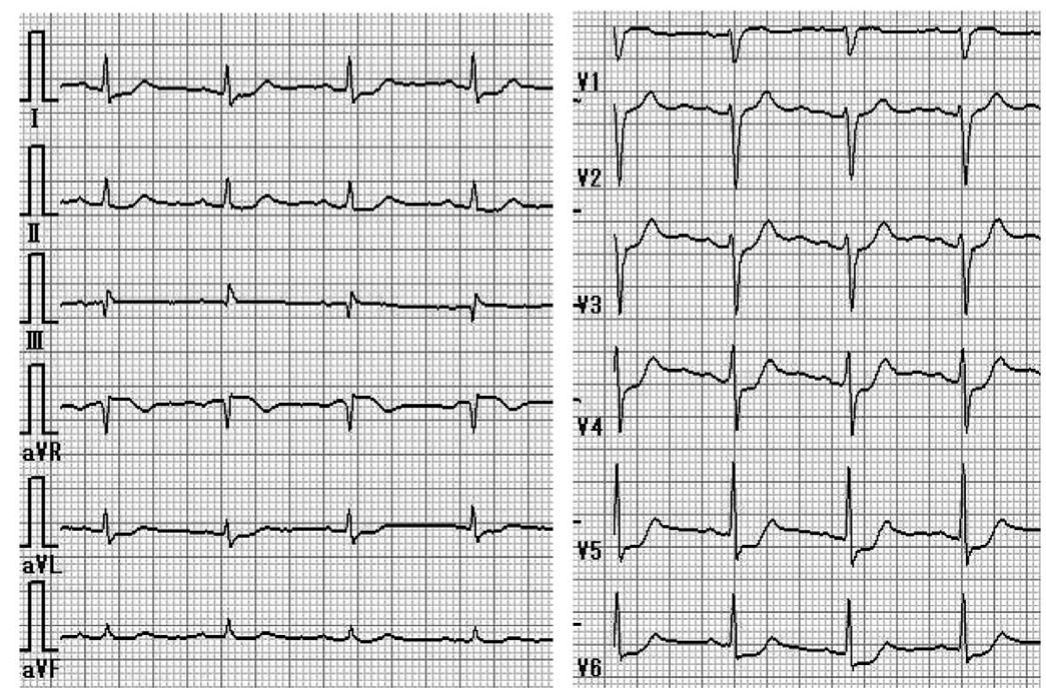

A

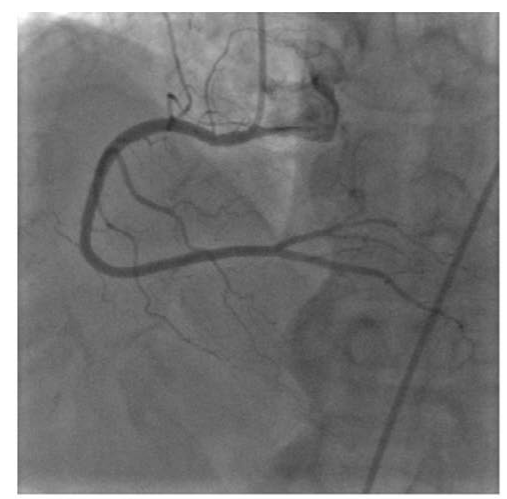

B

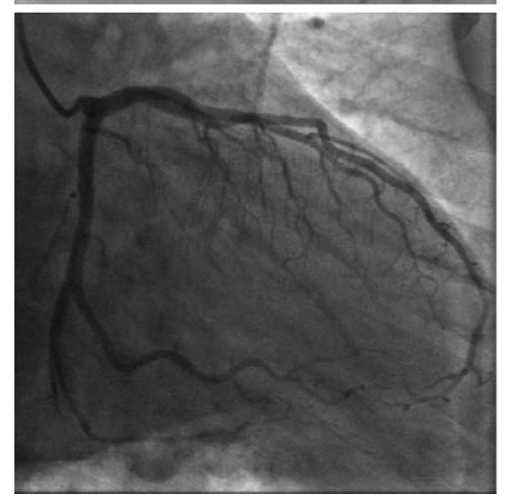

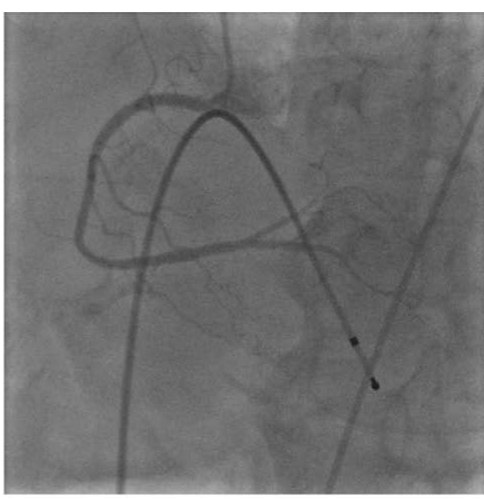

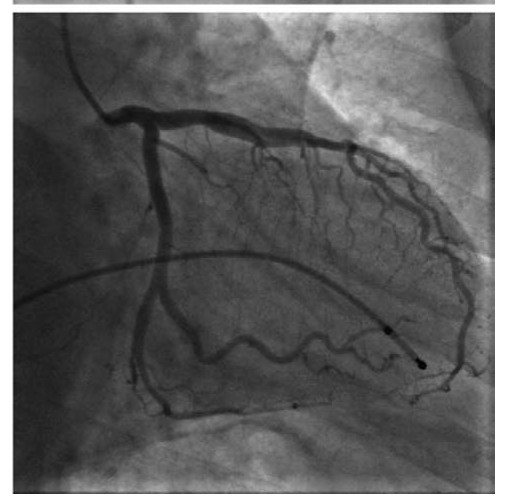

Figure 1. Electrocardiogram shows no STsegment depression at rest (A). Exercise treadmill test shows ST-segment depression in both limb leads and precordial leads $(\mathbf{B})$.
Figure 2. Coronary angiography shows no significant stenosis in either epicardial coronary artery (A). Provocative test with ergonovine does not induce any coronary artery spasm $(\mathbf{B})$. 

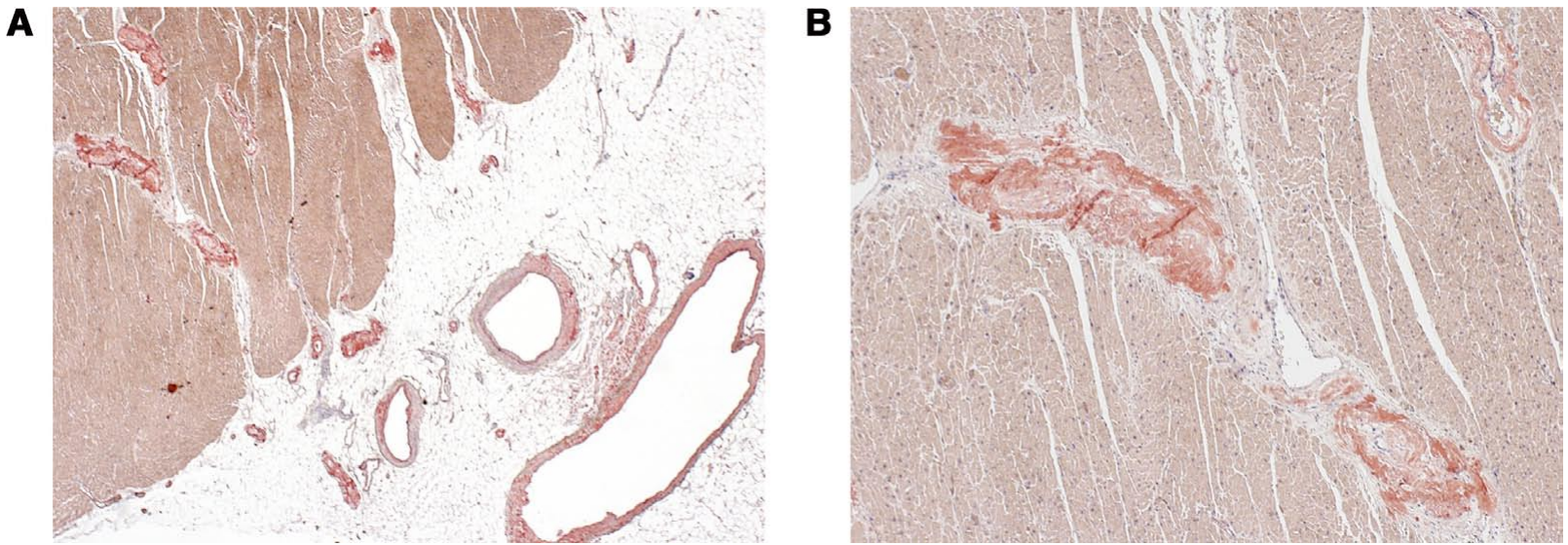

Figure 3. Amyloid stained with congo-red deposits within the walls of the intramyocardial arteries. There is little amyloid deposit within the walls of the epicardial coronary arteries or the myocardium (A). Some intramyocardial arteries are occluded with amyloid deposits $(\mathbf{B})$.

coronary arteries or the myocardium. No atherosclerosis was found in the epicardial coronary arteries, which was in line with the results of the previous series of coronary angiography.

\section{Discussion}

From a clinical and pathological point of view, this case is of paramount importance for the following reasons.

(1) Early clinical presentation of primary amyloidosis was typical angina symptoms, thus the patient was initially misdiagnosed as cardiac syndrome $\mathrm{X}$.

(2) Autopsy revealed accumulation of amyloid in the intramyocardial coronary arteries rather than the epicardial coronary arteries and myocardium itself.

(3) Congestive heart failure developed 8 years after the first episode of angina, which is a longer duration than previously reported.

Although congestive heart failure is the most common cardiac complication of primary amyloidosis, exertional angina caused by an intramyocardial coronary artery obstructed with amyloid depositions is rarely observed!-7 One report stated that preexisting anginal symptoms before the onset of congestive heart failure occurred in $17 \%$ of patients with primary amyloidosis, although accumulation of amyloid in the intramyocardial coronary arteries was detected in $66 \%$ of these patients? The present case is extremely valuable in terms of the accumulation of amyloid in the intracoronary arteries being accompanied by anginal symptoms as the early clinical presentation.

Suwaidi et al reported that congestive heart failure developed at 21 months after the onset of anginal symptoms in patients with cardiac amyloidosis? Other case reports ${ }^{10,11}$ describe the duration between the initial presentation of exertional angina and the later presentation of congestive heart failure as 3 and 7 years, respectively. In the present case, congestive heart failure developed 8 years after exertional angina occurred, which is longer than in those previous reports.

The diagnosis of primary amyloidosis in its early clinical stage is crucial, because some chemotherapeutic regimens are available for treating this disease ${ }^{12}$ Therefore, cardiac amyloidosis should be considered when the patient has severe angina without any significant stenoses of the epicardial coronary arteries on coronary angiography.

\section{Disclosure}

There is no financial or other relations that lead to a conflict of interest.

\section{References}

1. Smith RR, Hutchins GM. Ischemic heart disease secondary to amyloidosis of intramyocardial arteries. Am J Cardiol 1979; 44: 413417.

2. Narang R, Chopra P, Wasir HS. Cardiac amyloidosis presenting as ischemic heart disease: A case report and review of literature. Cardiology 1993; 82: 294-300.

3. Schafer S, Schardt C, Burkhard-Meier U, Heintzen MP, Strauer BE. Angina pectoris and progressive fatigue in a 61-year-old man. Circulation 1996; 94: 3376-3381.

4. Saffitz JE, Sazama K, Roberts WC. Amyloidosis limited to small arteries causing angina pectoris and sudden death. Am J Cardiol 1983; 51: $1234-1235$

5. Mueller PS, Edwards WD, Gertz MA. Symptomatic ischemic heart disease resulting from obstructive intramural coronary amyloidosis. Am J Med 2000; 109: 181-188.

6. Mesquita T, Chorão M, Soares I, Mello e Silva A, Abecasis P. Primary amyloidosis as a cause of microvascular angina and intermittent claudication. Rev Port Cardiol 2005; 24: 1521-1531.

7. Whitaker DC, Tungekar MF, Dussek JE. Angina with a normal coronary angiogram caused by amyloidosis. Heart 2004; 90: e54.

8. Neben-Wittich MA, Wittich CM, Mueller PS, Larson DR, Gertz MA, Edwards WD. Obstructive intramural coronary amyloidosis and myocardial ischemia are common in primary amyloidosis. Am J Med 2005; 118: 1287.

9. Suwaidi JA, Velianou JL, Getz MA, Cannon RO, Higano ST, Holmes $\mathrm{DR}$, et al. Systemic amyloidosis presenting with angina pectoris. Ann Intern Med 1999; 131: 838-841.

10. Yamano S, Motomiya K, Akai Y, Shiiki H, Toyama T, Hashimoto T, et al. Primary systemic amyloidosis presenting as angina pectoris due to intramyocardial coronary involvement: A case report. Heart Vessels 2002; 15: 157-160.

11. Ogawa H, Mizuno Y, Ohkawara S, Tsujita K, Ando Y, Yoshinaga $\mathrm{M}$, et al. Cardiac amyloidosis presenting as microvascular angina: A case report. Angiology 2001; 52: 273-278.

12. Kyle RA, Gertz MA, Greipp PR, Witzig TE, Lust JA, Lacy MQ, et al. A trial of three regimens for primary amyloidosis: Colchicine alone, melphalan and prednisone, and melphalan, prednisone, and colchicines. N Engl J Med 1997; 336: 1202-1207. 